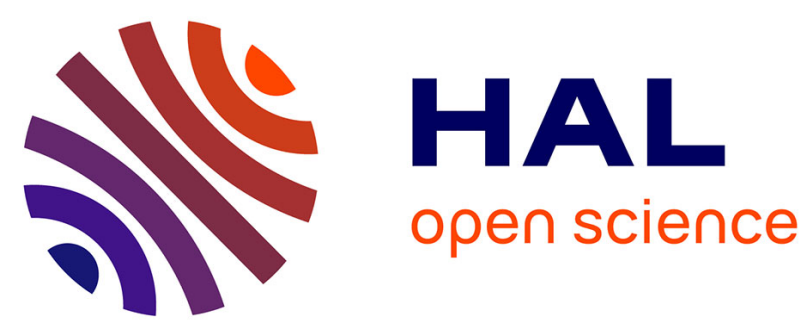

\title{
Analysis and Implementation of a Direct Phase Unwrapping Method for Displacement Measurement using Self-Mixing Interferometry
}

Ayesha Ehtesham, Usman Zabit, Olivier Bernal, Gulistan Raja, Thierry Bosch

\section{- To cite this version:}

Ayesha Ehtesham, Usman Zabit, Olivier Bernal, Gulistan Raja, Thierry Bosch. Analysis and Implementation of a Direct Phase Unwrapping Method for Displacement Measurement using Self-Mixing Interferometry. IEEE Sensors Journal, 2017, 17 (22), pp.7425-7432. 10.1109/JSEN.2017.2758440 . hal-01617743

\author{
HAL Id: hal-01617743 \\ https://hal.science/hal-01617743
}

Submitted on 17 Oct 2017

HAL is a multi-disciplinary open access archive for the deposit and dissemination of scientific research documents, whether they are published or not. The documents may come from teaching and research institutions in France or abroad, or from public or private research centers.
L'archive ouverte pluridisciplinaire HAL, est destinée au dépôt et à la diffusion de documents scientifiques de niveau recherche, publiés ou non, émanant des établissements d'enseignement et de recherche français ou étrangers, des laboratoires publics ou privés. 


\title{
Analysis and Implementation of a Direct Phase Unwrapping Method for Displacement Measurement using Self-Mixing Interferometry
}

\author{
Ayesha Ehtesham, Usman Zabit, Member, IEEE, Olivier D. Bernal Member, IEEE, Gulistan Raja, \\ Senior Member, IEEE, and Thierry Bosch, Senior Member, IEEE
}

\begin{abstract}
Self-Mixing (SM) or optical feedback interferometry has been widely used for displacement and velocity measurement applications. For metric information retrieval with $<\lambda / 2$ precision, various phase unwrapping methods have been proposed. However, these are computationally heavy and require large number of hardware resources, thereby hindering the development of real-time, embedded solutions for large bandwidth applications. In this regard, a simple and efficient feedback phase retrieval algorithm, called Consecutive Samples based Unwrapping (CSU) is presented. Detailed analysis of its error performance has been conducted as a function of key optical feedback parameters. A theoretical study has also been conducted to explain as to why such good error performance is obtained for such a simple algorithm by establishing a linear relation between the modulated laser power signal and the laser phase in the absence of optical feedback for specific ranges of key optical feedback parameters. We applied CSU on various simulated and experimentally acquired signals using SMI for the retrieval of harmonic and arbitrary displacements and found out that CSU retrieves target displacement with a precision of about $\lambda / 10$ while consuming much less time and hardware resources. The paper also presents FPGA based hardware design results of CSU and compares its performance with a traditional analytical phase unwrapping method in terms of maximum clock frequency, latency, and on-chip hardware resources. This hardware comparison strongly establishes the advantages of such a fast and computationally light algorithm, readily suitable for large bandwidth, embedded, real-time sensing applications.
\end{abstract}

Index Terms - Displacement Measurement; Self-Mixing; Optical Feedback Interferometry; Phase Unwrapping

\section{INTRODUCTION}

LASER Diode (LD) Self-Mixing (SM) or optical feedback interferometry (OFI) [1, 2] technique has been an active area of research during the last two decades for distance [3], flow [4], displacement [5], velocity [6], and vibration [7-9] sensing. As opposed to conventional interferometry, major advantage of SM interferometry is the simplicity and compactness of its setup (Fig. 1 shows a typical SM sensor setup) resulting in a self-aligned and cost-effective technique for sensing applications. However, recovery of displacement from the SM interferometric signal is not straight-forward due to its complex nature involving hysteresis [1] and non-linear fringes (with respect to target motion) (see Fig. 2). Thus, advanced

Date of publication: $2^{\text {nd }}$ of October 2017 algorithms are required to accurately measure the motion of remote target by using an SM sensor, as detailed below.

For displacement sensing, the SM signal can be processed using two major steps: fringe detection [10-12] and phase unwrapping [13-16]. In the absence of strong feedback [2, 17, 18], each fringe corresponds to a target displacement of $\lambda_{0} / 2$ in the first order approximation, where $\lambda_{0}$ is laser wavelength without feedback in both the external and internal laser cavities [19]. To improve this basic resolution, various phase unwrapping techniques exist [11, 13-15, 18, 20-23] to unwrap the laser feedback phase leading to accuracies from $\lambda_{0} / 8$ to $\lambda_{0} / 60$. Let us look into some of the major such techniques.

Based on the seminal model of optical feedback by Lang and Kobayashi [24], displacement retrieval from a weak feedback regime SM signal was achieved [13] by unwrapping the laser feedback phase. It used pre-calibrated values of two fundamental SM parameters: line-width enhancement factor $\alpha$ and optical feedback coupling factor $C$ [25], defined later in the paper. The method is claimed to have measurement error of $<50 \mathrm{~nm}$ in displacement reconstruction for laser wavelength of $673 \mathrm{~nm}$, resulting in an accuracy of about $\lambda_{0} / 13$.

Further improvement was achieved by an auto-adaptive SM retrieval algorithm, called as the phase unwrapping method (PUM), for moderate feedback regime [14]. It consists of two major steps: 1) rough estimation of laser phase under feedback $\Phi_{\mathrm{F}}(t)$ and 2) joint estimation of $C, \theta=\arctan (\alpha)+\Phi_{\mathrm{F}}(0)$, and displacement $D(t)$, where $\Phi_{\mathrm{F}}(0)$ represents the initial laser phase under feedback. The proposed method increases the accuracy of the SM sensor to $\lambda_{0} / 16$.

The results of PUM were then further improved in [15] by identifying an error in previous methods i.e. local feedback phase inversions. Similar identification and improvement of PUM was proposed in [16] by carrying out a detailed theoretical analysis of PUM, resulting in the so called Improved Phase Unwrapping Method (IPUM), leading to even better accuracy of about $\lambda_{0} / 40$. The approach uses detection of peaks and valleys of an SM signal, followed by application of a specifically designed arcos function on different SM signal segments, as identified by the peaks and the valleys, resulting in correct retrieval of $\Phi_{\mathrm{F}}(t)$. Moreover, this paper also highlights that the normalization of an SM signal needs much more care as it directly affects the displacement retrieval [16].

Although the PUM and the IPUM give considerably good accuracy for the LD based SM sensors, yet these algorithms are computationally very heavy and require large number of 


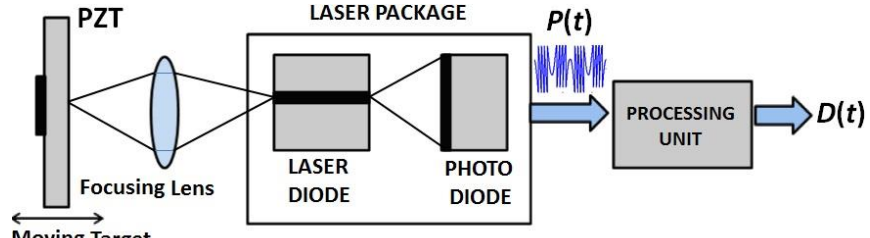

Moving Target

Fig. 1: Schematic diagram of self-mixing laser sensor for displacement measurement requiring only a laser package and a lens. A piezoelectric transducer (PZT) has been used as remote target. Variations in the optical output power $P(t)$ are processed by a computing unit e.g. a computer or an FPGA device in order to retrieve the target displacement $D(t)$.

hardware resources. Moreover, these algorithms use long iterative routines [26] for the estimation of $C$ and $\alpha$ parameters which makes them inefficient for real-time, high bandwidth, embedded sensing applications.

Another SM approach with slightly poorer accuracy in displacement measurement but with much faster signal processing capability involves direct unwrapping of the SM signal fringes to reconstruct $D(t)$. Such an approach [22], valid only for the moderate feedback regime $(1<C<4.6)$, comprised of all analog circuits and made use of an apparent linearity of the SM signal fringes for $C>1$. A similar approach [27] used a Digital Signal Processor (DSP) for SM signal processing, again under moderate feedback regime. This method [27], however, depends upon various assumptions, as detailed below. Firstly, the method assumes that for $C>1$, the function $\cos \left(\Phi_{F}(t)\right)$ is linearly dependent on the target motion, resulting in a claimed measurement accuracy of $50 \mathrm{~nm}$ for $C>1$ for $\lambda_{0}=780 \mathrm{~nm}$ i.e. $\lambda_{0} / 15$. However, the present work shows that such an assumption is only valid for a restricted $C$ range within moderate feedback regime, beyond which measurement accuracy becomes far poorer than $\lambda_{0} / 15$. Secondly, in order to compensate the phase discontinuity associated with each SM fringe, this approach uses a scaling factor which is dynamically adjusted by using previous fringe discontinuity amplitude. Such a configuration, however, results in high error just after each direction reversal. This increased error occurs because of the marked hysteresis associated with all SM signals belonging to $C>1.5$. On the other hand, the present work does not suffer from such an error. Thirdly, although it [27] processes both weak and moderate feedback regime signals, yet the employed signal processing is vastly different for weak feedback regime which is just made up of fringe counting and does not use SM direct unwrapping. This is in opposition to the present work which employs direct unwrapping for all major optical feedback regimes. Lastly, both methods [22, 27] make use of standard low pass filtering (LPF) in order to reduce the previously mentioned errors by filtering out the high frequency discontinuities present within unwrapped or retrieved displacement at the cost of reduced sensing bandwidth. The studies conducted in the present paper, however, do not use such LPF so as to explicitly detail the high frequency natured errors that appear during this direct SM unwrapping approach.

In this context, a simple and real-time natured algorithm namely, Consecutive Samples based Unwrapping (CSU) has been proposed in [20]. The fast real-time nature of this algorithm arises from the fact that by introducing a delay of one sample, it requires only two consecutive samples of SM signal to detect any SM fringe while continuously updating the corresponding displacement output as compared to the complex iterative optimization routines of the PUM for the joint estimation of $C$ and $\alpha$.

Although the accuracy of CSU is about $\lambda_{0} / 10$ [20] which is slightly less as compared to the $\lambda_{0} / 16$ accuracy of PUM, but, the real-time nature and simplicity of this method makes it very attractive for its application on real-time, large bandwidth signals such as autonomous, embedded sensing of ultrasonic vibrations [28]. Moreover, CSU provides correct unwrapping of SM signal belonging to major feedback regimes, covering a wide range of $C$, with satisfactory error results, as opposed to the direct unwrapping approaches of $[22,27]$.

In this paper, a detailed analysis of the CSU algorithm is presented for $C \in[0.5,7]$, thus spanning weak, moderate, and strong feedback regimes while $\alpha \in[1,10]$, thereby covering typical LDs employed in SM sensors. A theoretical study has been conducted to explain as to why such a simple SM algorithm provides such good error performance for a specific range of optical feedback coupling. CSU algorithm has been tested on various simulated and experimental SM signals belonging to major feedback regimes. Lastly, FPGA based implementation results of CSU and PUM demonstrate that CSU consumes much less time and hardware resources as compared to analytical phase unwrapping methods [14-16].

The rest of the paper is organized as follows: Section II gives a brief review of the basic theory of self-mixing followed by Section III which elaborates the basic methodology of the CSU algorithm. A mathematical base to support the CSU approach has been established in Section IV whereas detailed simulation and experimental results of CSU have been presented in Section V and Section VI respectively. Section VII provides a detailed comparison of FPGA based hardware implementation of CSU and PUM, demonstrating the usefulness of CSU for real-time processing of high bandwidth, embedded sensing applications. The paper ends with Discussion and Conclusion.

\section{THEORY OF SELF-MIXING}

The theory of SM interferometry has been elaborated in different references $[1,2,24]$ and is summarized below.

Let $D(t)$ represent the instantaneous distance between the LD driven by a constant injection current with highly stabilized temperature and a remote surface that back-scatters a small amount of optical power back into the LD cavity. Under this optical feedback phenomenon, the free running laser wavelength $\lambda_{0}$ is slightly modified into $\lambda_{\mathrm{F}}(t)$ and varies with $D(t)$. The wavelength fluctuations can be found by solving the so-called excess phase equation [24]:

$$
\Phi_{0}(t)=\Phi_{F}(t)+C \sin \left[\Phi_{F}(t)+\arctan (\alpha)\right]
$$

where $\Phi_{\mathrm{F}}$ and $\Phi_{0}$ represent two phase signals (with and without feedback, respectively) which can be written as a function of the wavelengths $\lambda_{\mathrm{F}}(\mathrm{t})$ and $\lambda_{0}$, respectively:

$$
\begin{aligned}
& \Phi_{F}(t)=2 \pi \frac{D(t)}{\lambda_{F}(t) / 2}=2 \pi v_{F}(t) \tau(t) \\
& \Phi_{0}(t)=2 \pi \frac{D(t)}{\lambda_{0}(t) / 2}=2 \pi v_{0}(t) \tau(t)
\end{aligned}
$$

where $\tau(t)=2 D(t) / c$ is the round-trip time, $c$ is the speed of light in vacuum and $v_{\mathrm{F}}(t)$ and $v_{0}(t)$ represent the optical frequencies with and without optical feedback, respectively. 


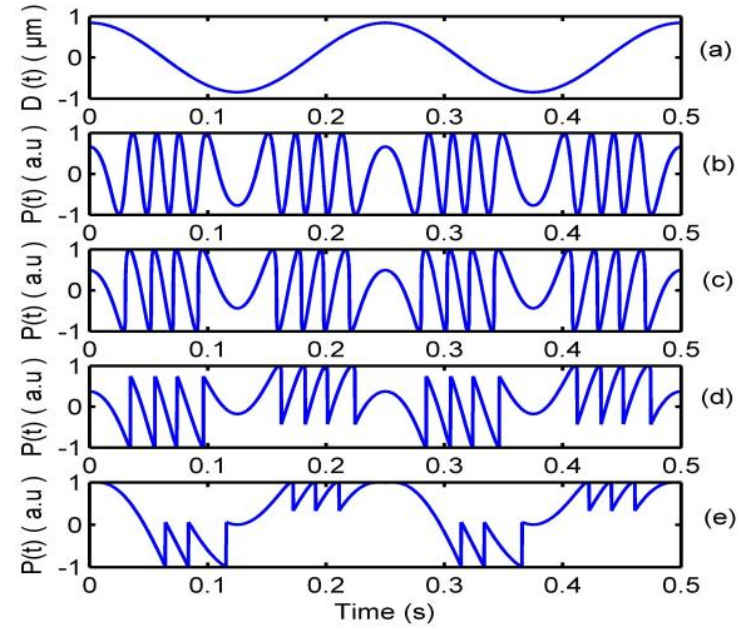

Fig. 2: (a): Target displacement; and normalized SM signals simulated by [29] for $\alpha=5$ and (b) $C=0.2$, (c) $C=1$, (d) $C=2.4$ and (e) $C=5.5$.

The value of $\Phi_{\mathrm{F}}(t)$ can be extracted from the optical output power (OOP) of the laser diode $P(t)$ using

$$
P(t)=P_{0}\left[1+m \cos \left[\Phi_{F}(t)\right]\right]
$$

where $P_{0}$ is laser power without feedback and $m$ a modulation index. If $P_{N}(t)$ represents the normalized OOP, then [29]:

$$
P_{N}(t)=\cos \left(\Phi_{F}(t)\right)
$$

The Coupling factor $C$ depends on LD parameters but also on both $D(t)$ and surface reflection coefficient $R_{t}$ [13]:

$$
C=\frac{\tau_{D}}{\tau_{L}} \gamma \sqrt{1+\alpha^{2}} \mathrm{~K}_{\text {ext }}
$$

where $\tau_{\mathrm{L}}$ and $\tau_{\mathrm{D}}$ are the round trip times in the internal and external cavities respectively, $\gamma$ the coupling efficiency and $\kappa_{e x t}$ depends on the surface reflectivity of the target.

$C$ is a fundamental parameter in SM interferometry as it significantly determines the shape of the OOP signal (see Fig. 2 ). Based on the value of $C$, the OOP signal can be classified into the following three major feedback regimes:

1. Weak feedback regime: the value of $C$ such that $0.1<C$ $<1$ represents the weak optical feedback regime with the OOP signal characterized by sinusoidal or asymmetric SMI fringes without any sharp discontinuities [26].

2. Moderate feedback regime: the value of $C$ such that $1<$ $C<4.6$ represents the moderate optical feedback regime [14] with the OOP signal characterized by saw-tooth like SM fringes which also exhibit hysteresis [30].

3. Strong feedback regime: the value of $C>4.6$ represents strong feedback regime [31], characterized by a chaotic SM signal $[2,17]$ including fringe-loss $[18,29]$.

As the value of $C$ increases, hysteresis in SM signal increases while the height of SM fringes decreases and fringes begin to disappear for $C>4.6$ (See Fig. 2) leading to a completely fringe-less SM signal for very strong feedback coupling $[18,29]$.

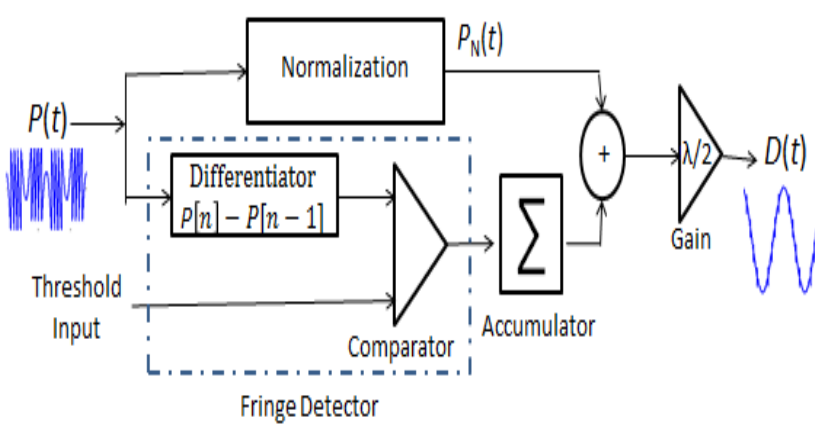

Fig.3: Schematic block diagram of the steps involved in CSU.
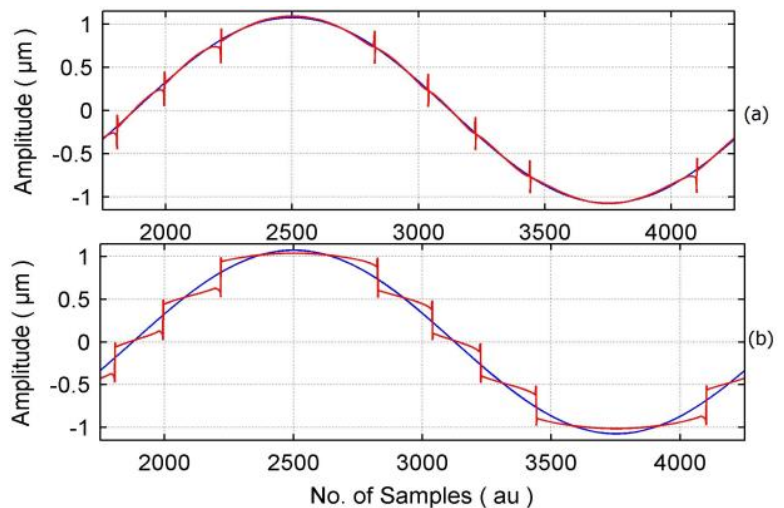

Fig. 4 (a): CSU based displacement (red) versus reference target displacement $D(t)=\Phi_{0}(t) * \lambda / 4 \pi$ (blue); (b): $\Phi_{\mathrm{F}}(t)$ based displacement (red) versus reference target displacement (blue) for $\mathrm{C}=1.8$ and $\alpha=5$.

\section{CONSECUTIVE SAMPLES BASED UNWRAPPING}

CSU algorithm is based on the assumption that, for specific $[C, \alpha]$ ranges, the shape of SM signal represents the actual target movement except at the discontinuities occurring in SM signal for every $\lambda / 2$ displacement [20,22, 27]. Therefore, by correctly detecting and unwrapping these discontinuities, a signal that represents the actual target movement can be obtained. A schematic block diagram of the CSU approach is shown in Fig. 3.

The CSU algorithm processes the input signal in a manner very similar to that of first step of PUM. As previously mentioned, in the first step of PUM, roughly estimated feedback phase $\widehat{\Phi}_{F}(t)$ is obtained. This is achieved by taking $\operatorname{arcos}$ of $P_{\mathrm{N}}(t)$ as per (5). This results in a feedback phase which is wrapped between $[0 \pi]$ due to use of arcos function. This wrapped feedback phase is then unwrapped by adding or subtracting $2 \pi$ at each fringe discontinuity (depending upon whether the fringe has a rising or falling discontinuity). Then, in second step, $\Phi_{F}^{\wedge}(t)$ is used to estimate $\Phi_{0}(t)$ by solving (1) after conjoint estimation of $\mathrm{C}$ and $\alpha$ [26]. The linear relation between $\Phi_{0}(t)$ and $D(t)$, as per (3), then provides target motion. 


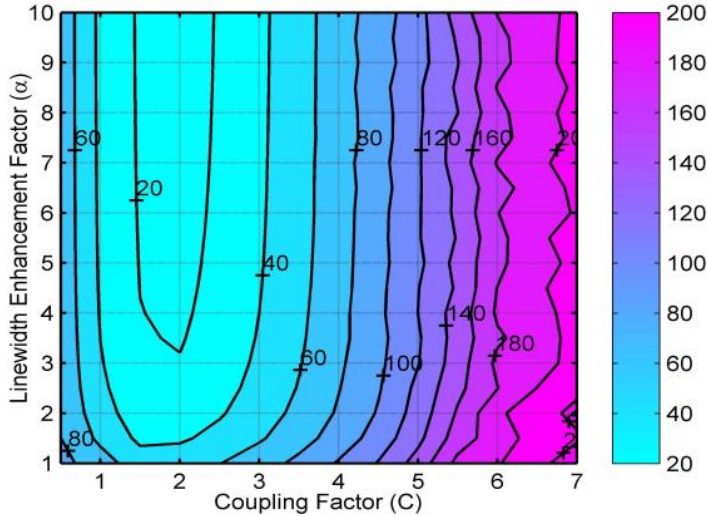

Fig. 5: RMS error $\epsilon_{\mathrm{RMS}}$ results (nm) of CSU for noise-less simulated SM signals for varying values of $\mathrm{C}$ and $\alpha$ corresponding to $2.5 \mu \mathrm{m}$ sinusoidal target displacement with $\lambda=785 \mathrm{~nm}$.

Similarly, the CSU algorithm unwraps the normalized SM power signal $P_{\mathrm{N}}(t)$ (so that $P_{\mathrm{N}}(t) \in\left[\begin{array}{ll}-1 & 1\end{array}\right]$ ) by unwrapping its discontinuities. These fringe discontinuities are detected by a fringe detector (see Fig. 3) by comparing the derivative of $P(t)$ to a threshold. Each fringe is then assigned 1 or -1 depending upon the rising and falling discontinuity of the fringe. An accumulation of all detected fringes results in a stair-case signal due to addition or subtraction of 2 at each fringe discontinuity. A direct addition of stair-case signal to $P_{\mathrm{N}}(t)$ then leads to the unwrapped $P_{N}(t)$ which is directly proportional to $D(t)$ under the assumption that each fringe is a linear approximation of the actual target displacement. This assumption eliminates the requirement of $C$ and $\alpha$ estimations, thereby rendering CSU much simpler, yet precise for specific $[\mathrm{C}, \alpha]$ values (as detailed below), and easier to implement for real-time, high bandwidth applications.

Next section mathematically establishes the important linearity between normalized unwrapped $\mathrm{PN}(\mathrm{t})$ and $\mathrm{D}(\mathrm{t})$, thereby validating this assumption for specific ranges of [C, $\alpha$ ]. This assumption is also supported by Fig. 4 (a) showing that the unwrapped $\mathrm{P}(\mathrm{t})$ signal based displacement (CSU output) better resembles the reference target displacement $\mathrm{D}(\mathrm{t})=\Phi_{0}(\mathrm{t}) * \lambda / 4 \pi$ whereas $\Phi_{F}(t)$ based displacement (output of first step of PUM) poorly resembles the reference target displacement, (see Fig. 4 (b)).

Displacement retrieval performance of CSU is presented in Fig. 5 as a function of fundamental SM parameters of $C$ and $\alpha$ such that $C \in[0.5,7]$, thus spanning weak, moderate, and strong feedback regimes while $\alpha \in[1,10]$, thereby covering typical LDs employed in SM sensors. Fig. 5 thus represents the values of RMS errors calculated by comparing the CSU retrieved displacement with the simulated sinusoidal reference target displacement of $2.5 \mu \mathrm{m}$ for $\lambda_{0}=785 \mathrm{~nm}$ without addition of any noise in the SM signal. Note that for restricted range of $C \in[1.5,2.5]$ and $\alpha>3$, the accuracy of CSU is about $20 \mathrm{~nm}$ which is better than that of PUM for $\lambda_{0}=785 \mathrm{~nm}$ [14]. Furthermore, for $C \in[1,3]$ and $\alpha>2$, CSU provides an accuracy of about $40 \mathrm{~nm}$, which is very similar to that of PUM (a)
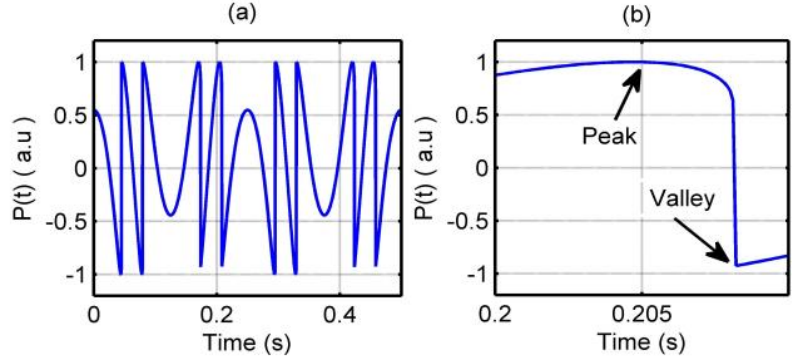

Fig. 6: Fringe fold-back phenomenon (local amplitude reversal) in an SM signal with $\alpha=5$ and $C=1.5$. (b) Zoomed portion indicating fringe foldback delimited between local fringe peak and valley locations.

for $\lambda_{0}=785 \mathrm{~nm}$. It thus shows that high measurement accuracy is provided by CSU approach for restricted $\mathrm{C}$ and $\alpha$ ranges while using a much simpler algorithm than PUM.

\section{THEORETICAL ANALYSIS OF CSU APPROACH}

CSU based target displacement is reconstructed by adding the unwrapped discontinuities (each corresponding to a $\lambda / 2$ displacement) to the instantaneous normalized SM signal. Nevertheless, due to the inherent simplicity of the CSU, the reconstruction is subject to errors directly related to both the size and shape of SM fringes (as graphically presented in the next section). It is also evident from Fig. 5 that minimum CSU displacement reconstruction RMS error $\epsilon_{\mathrm{RMS}}$ can be reached for $C \in[1.5,2.5]$.

Here, the following analysis aims to explain the origins of such inherent accuracy while using a simplistic algorithm. First, in order to add the unwrapped discontinuities with the instantaneous SM signal, the SM signal should be normalized to get $P_{\mathrm{N}}(t)$ in order to reduce error as much as possible. As a result, such an SM signal should be preferably a class I, II or III as described in [30] since the normalization process does not introduce any inherent error for these classes.

Further, in the case of a complete SM fringe, the fringe fold-back before the phase discontinuity should be reduced as much as possible as it would be wrongly interpreted by the CSU as a change of direction before the discontinuity. (Fringe fold-back, delimited by the local maximum and minimum SM fringe amplitudes usually denoted as peak and valley locations respectively [15-16], is illustrated in Fig. 6 (b) in which local amplitude reversal [15-16] is seen before each fringe discontinuity.) In addition, at first CSU was dedicated for the moderate feedback regime signals as SM signal fringes can be determined by detecting discontinuities. These discontinuities arise from the loss of bijectivity of eq. (1) and it was shown in [29] that they occur whenever $\Phi_{\text {F }}$ presents infinite slopes [29]:

$$
\begin{gathered}
\Phi_{F, R}=k \pi-\arctan (\alpha)+\beta \\
\Phi_{F, F}=(k+2) \pi-\arctan (\alpha)-\beta
\end{gathered}
$$

where $\Phi_{F, R}$ and $\Phi_{F, F}$ represents the feedback phase at rising and falling discontinuities respectively, $k$ is an even integer and $\beta=\operatorname{arcos} \frac{-1}{C}$. As a result, $\Phi_{F, R}$ and $\Phi_{F, F}$ should be as close as possible to 0 and $\pi$ respectively. 

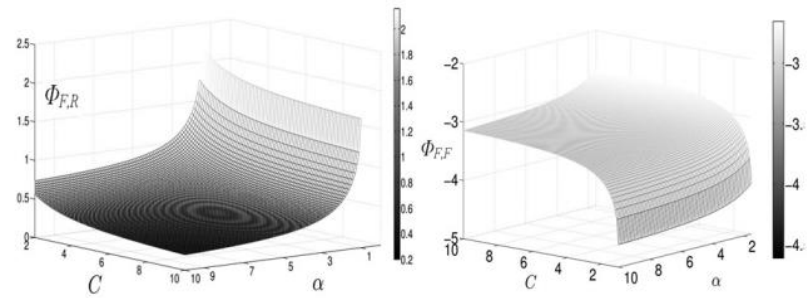

Fig. 7: (a): Graphical representation of the phase value $\Phi_{\mathrm{F}}$ before the rising discontinuity $\Phi_{\mathrm{F}, \mathrm{R}}$ versus $(\mathrm{C} ; \alpha)$ for $(\mathrm{C} ; \alpha) \in([1,10] ;[1,10]) ;(\mathrm{b})$ : Graphical representation of the phase value $\Phi_{\mathrm{F}}$ before the falling discontinuity $\Phi_{\mathrm{F}, \mathrm{F}}$ versus $(\mathrm{C} ; \alpha)$ for $(\mathrm{C} ; \alpha) \in([1,10] ;[1,10])$.

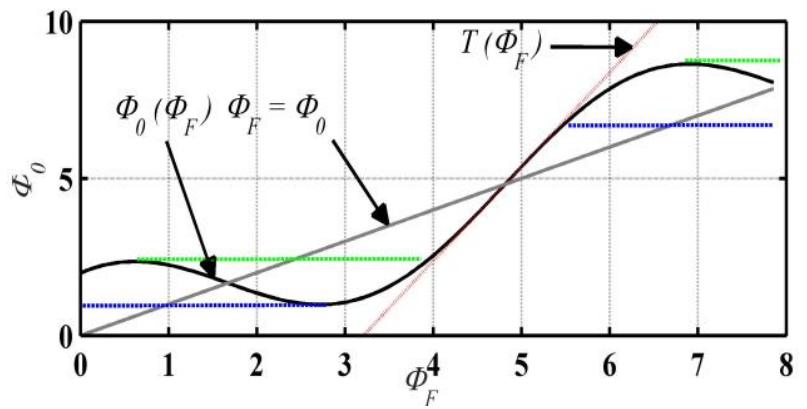

Fig. 8: Graphical representation of the phase value $\Phi_{0}$ before the rising discontinuity versus $\Phi_{\mathrm{F}}$ for $(\mathrm{C} ; \alpha)=[2,10]$ in black line with its tangent (dotted red line) at the intersection between the black curve and the light grey line corresponding to $\Phi_{0}=\Phi_{\mathrm{F}}$ (dotted green line shows the rising discontinuity while dotted blue line shows the falling discontinuity)

Fig. 7 (a) and Fig. 7 (b) clearly show that the higher the $C$ and $\alpha$ values, the closer $\Phi_{F, R}$ and $\Phi_{F, F}$ are from 0 and $\pi$ respectively as it will reduce the error induced by the fringe fold-back (if any) before the discontinuity (a small fringe fall back can be observed for the rising fringe only in Fig.4 (a) for $C=1.8$ ). However, the higher the $C$, the smaller the fringe is (see Fig. 2) which induces discontinuities in the reconstructed displacement (as graphically presented in the next section). Therefore, $C \in[1.5,2.5]$ provides a good tradeoff.

Even though the CSU error is maximum around the phase discontinuities, still its reconstruction performance is relatively good compared to the required amount of computational power. To explain this aspect, it is interesting to note that the higher the $\alpha$ value, the better the CSU performance is. Actually, for high $\alpha$ value, $\arctan (\alpha) \approx \pi / 2$. As a result, for high $\alpha$ value, (1) can be expressed as:

$$
\Phi_{0}(t)=\Phi_{F}(t)+C \cos \left(\Phi_{F}(t)\right)
$$

At this point, it is interesting to note that the optical output power is modulated by $\cos \left(\Phi_{\mathrm{F}}(t)\right)$, as seen in (3). Hence, (9) can thus be rewritten as:

$$
\cos \left(\Phi_{F}(t)\right)=\frac{\Phi_{0}(t)-\Phi_{F}(t)}{C}
$$

Consequently, it is interesting to note from (10) that under the assumption $\Phi_{F}$ is approximately proportional to $\Phi_{0}\left(\Phi_{F}=\right.$ $\left.\gamma \Phi_{0}\right)$, the modulated output power becomes directly proportional to the target displacement:

$$
\cos \left(\Phi_{F}(t)\right)=\frac{1-\gamma}{C} \Phi_{0}(t)
$$

Therefore, using (5) and (11), it can be stated as:

$$
P_{N}(t)=\rho \Phi_{0}(t)
$$

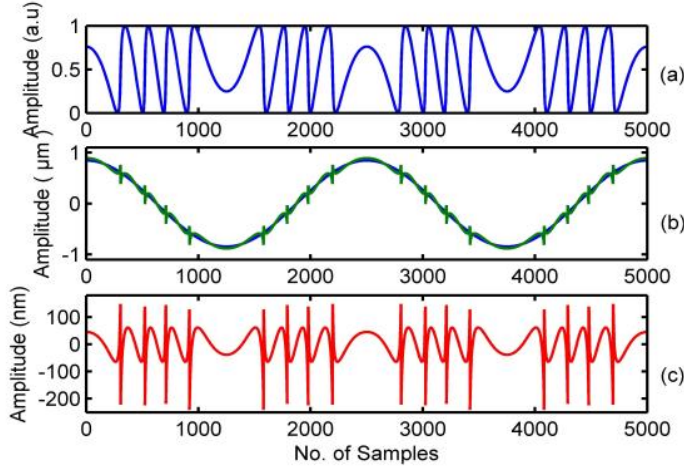

Fig. 9: Displacement reconstruction using CSU for $\mathrm{C}=0.8, \alpha=5$ and $\lambda$ $=785 \mathrm{~nm}$; (a): Normalized SM signal; (b) Reconstructed displacement using CSU (green) with reference displacement (blue); (c) Error between reference and reconstructed displacement.

where $\rho=\frac{1-\gamma}{C}$. It can now be stated that:

$$
P_{N}(t)=\frac{4 \pi \rho}{\lambda} D(t)
$$

This is a fundamental relationship indicating that if $\Phi_{\mathrm{F}}$ is proportional to a certain extent to $\Phi_{0}$ then for high $\alpha$ values, proportionality exists between the normalized SM signal and the actual target motion. This assumption serves as the basis for the CSU algorithm, for specific $C$ and $\alpha$ range. In addition, this proportionality of (11) can be observed by the tangent $T$ at the point where $\Phi_{0}=\Phi_{F}=k \pi-\arctan (\alpha)$ which equation is expressed as:

$$
T\left(\Phi_{F}\right)=(-1)^{k+1} C(k \pi-\arctan (\alpha))+\left(1+(-1)^{k} C\right) \Phi_{F}(t)
$$

Fig. 8 clearly shows that such linearity exists between $\Phi_{0}$ and $\Phi_{F}$ along the tangent at the curve $\Phi_{0}\left(\Phi_{F}\right)$ at $\Phi_{0}=\Phi_{F}=$ $k \pi-\arctan (\alpha)$. Specifically, such a linear approximation assumption is correct around the mid-point of each SM fringe (as seen in Fig. 9 in which maximum correlation between $\Phi_{0}\left(\Phi_{F}\right)$ and $T\left(\Phi_{F}\right)$ is seen in the zone delimited by rising and falling fringe discontinuity locations, indicated by dotted green and dotted blue horizontal lines respectively) but not so at the SM fringe extrema, as also evidenced by the introduction of error in CSU output around each fringe discontinuity (as seen in the next section).

\section{Simulated RESUltS}

A detailed error analysis of CSU has already been presented in Fig. 5 for $C \in[0.5,7]$, and $\alpha \in[1,10]$. Some of these results are presented below for illustrative purposes.

\section{A. Displacement Retrieval for SM Weak Feedback Regime}

Fig. 9 presents CSU performance for a weak feedback regime SM signal with $C=0.8$ and $\alpha=5$. The RMS error of this case is $49.23 \mathrm{~nm}$ whereas the peak error is $240.3 \mathrm{~nm}$ occurring around the discontinuity as expected.

\section{B. Displacement Retrieval for Moderate Feedback Regime}

Fig. 10 shows a moderate feedback regime SM signal with $C=1.8$ and $\alpha=5$. The RMS error of this case, presented in Fig. 10 (c) is $19.25 \mathrm{~nm}$ whereas peak error is $209.4 \mathrm{~nm}$. 


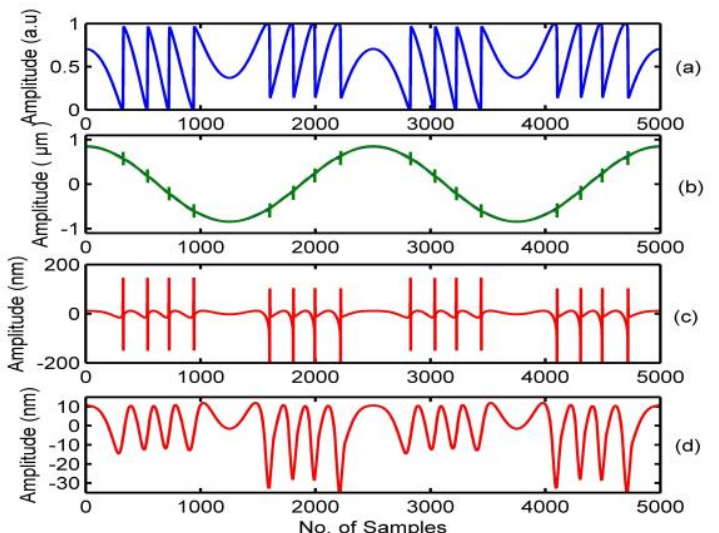

Fig. 10: CSU based $D(t)$ retrieval for $\mathrm{C}=1.8, \alpha=5$ and $\lambda=785 \mathrm{~nm}$; (a): Normalized SM signal; (b) Reconstructed $D(t)$ using CSU (green) with reference $D(t)$ (blue); (c) Error between reference and reconstructed $D(t)$. (d) Error between reference and low pass filtered (cut-off frequency $=$ $160 \mathrm{~Hz}$ for sampling frequency $=10 \mathrm{KHz}$ ) reconstructed $D(t)$.

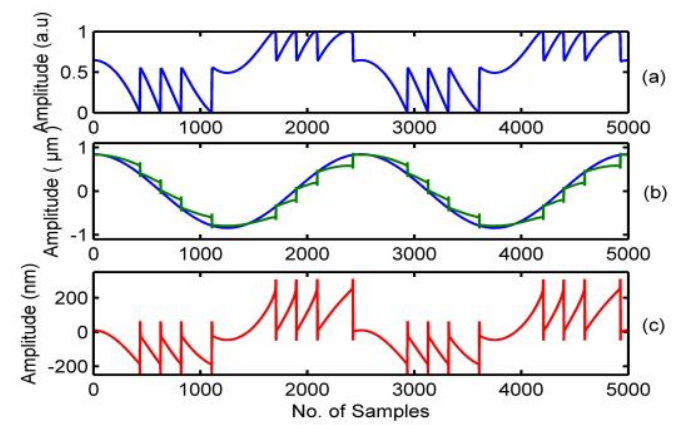

Fig. 11: Displacement reconstruction using CSU for $\mathrm{C}=5.0, \alpha=5$ and $\lambda=$ $785 \mathrm{~nm}$; (a) SM signal ; (b) Reconstructed $D(t)$ using CSU (green) and Reference Displacement (blue); (c) Error in reconstructed displacement.

\section{Displacement Retrieval for SM Strong Feedback Regime}

Fig. 11 shows a strong feedback regime SM signal with $C=$ 5.0 and $\alpha=5$ processed by CSU, resulting in an RMS error of $115.08 \mathrm{~nm}$ and a peak error of about $315.4 \mathrm{~nm}$.

It may be highlighted that all the results presented here have not undergone any filtering in order to ensure that actual performance of CSU algorithm for major SM regimes can be ascertained. However, CSU error can be significantly reduced $[20,22]$, as shown in Fig. 10 (d), at the cost of reduced bandwidth, by incorporating a low pass filter which removes the sharp discontinuities that exist in CSU output, caused due to phase change at each SM fringe transition.

\section{Displacement with Fractional Half-wavelength Amplitude}

An interesting property of CSU is that in addition to recovering the target vibrations with peak-to-peak (p-p) amplitude $>\lambda_{0} / 2$, it can also recover the target vibrations with $\mathrm{p}$-p amplitude of $<\lambda_{0} / 2$ if such vibration is superimposed on a vibration $>\lambda_{0} / 2$. This property makes CSU better than the approaches used in $[11,19,32]$ as these approaches cannot recover the target vibration with p-p amplitude $<\lambda_{0} / 2$. Fig. 12 depicts such a case with the simulated displacement of $D(t)=$ $(8 \lambda) \sin (2 \pi 10 t)+(\lambda / 4) \sin (2 \pi 40 t)$ for $C=1.8, \alpha=5$ and $\lambda_{0}=$ $785 \mathrm{~nm}$. Corresponding SMI signal is also plotted along with the reconstructed displacement using CSU. The RMS error for this case is $33.17 \mathrm{~nm}$ and peak error is $207.2 \mathrm{~nm}$.

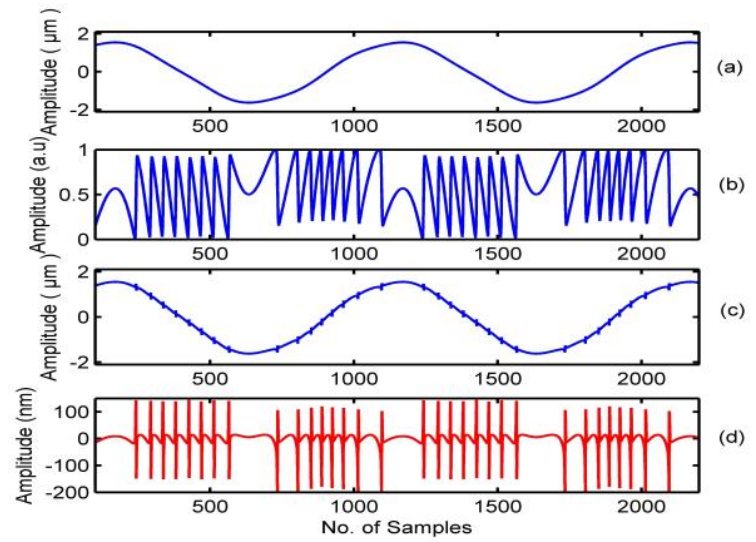

Figure 12: Recovery of target motion including segments with peak-topeak amplitude of $<\lambda_{0} / 2$ (a): Reference Displacement; (b): SM signal; (c): Reconstructed $D(t)$; (d): Error in nm.

\section{EXPERIMENTAL RESULTS}

The CSU algorithm is tested on several experimentally acquired SM signals using a PZT (Piezo-electric Transducer) from Physik Instrumente ${ }^{\circledR}$ as the target displacement (see Fig. 1). The PZT is equipped with a built-in capacitive sensor of 2 $\mathrm{nm}$ measurement precision, used as a reference to measure error performance of CSU. The SM sensor is based on LD package from Sanyo ${ }^{\circledR}$ (DL7140) with $\lambda_{0}=785 \mathrm{~nm}$, output power of $60 \mathrm{~mW}$, and threshold current of $50 \mathrm{~mA}$.

The results obtained after application of CSU algorithm on several experimentally acquired SM signals are presented in Table I. Performance of PUM, also using a LPF, is also presented for the sake of comparison. Note that the 13th and 14th acquisitions of Table I are of high feedback regime SM signals with one lost fringe in both cases [18]. Fig. 13 and Fig. 14 graphically present experimental results of 10th and 15th acquisition respectively, where the latter is of an experimental SM signal corresponding to arbitrary target motion.

Table I

COMPARISON OF MAXIMUM \& RMS ERRORS FOR CSU \& PUM USING EXPERIMENTALLY ACQUIRED SM SIGNALS

\begin{tabular}{|c|c|c|c|c|c|c|c|}
\hline \hline No & $\begin{array}{c}\text { Estim } \\
\text { ated } C \\
\text { value } \\
\text { by } \\
\text { PUM }\end{array}$ & $\begin{array}{c}\text { Amplitud } \\
\text { e of } \\
\text { vibrating } \\
\text { target } \\
(\mu \mathrm{m})\end{array}$ & $\begin{array}{c}\text { Targe } \\
\mathrm{t} \\
\text { Frequ } \\
\text { ency } \\
(\mathrm{Hz})\end{array}$ & $\begin{array}{c}\text { RMS } \\
\text { Error } \\
\text { of } \\
\text { CSU } \\
(\mathrm{nm})\end{array}$ & $\begin{array}{c}\text { Max. } \\
\text { Error } \\
\text { of } \\
\text { CSU } \\
(\mathrm{nm})\end{array}$ & $\begin{array}{c}\text { RMS } \\
\text { Error } \\
\text { of } \\
\text { PUM } \\
(\mathrm{nm})\end{array}$ & $\begin{array}{c}\text { Max. } \\
\text { Error of } \\
\text { PUM } \\
{[14]} \\
(\mathrm{nm})\end{array}$ \\
\hline 1 & 0.91 & 1.20 & 40 & 59.3 & 211.3 & 30.6 & 114.5 \\
\hline 2 & 1.27 & 1.65 & 50 & 49.2 & 212.5 & 55.5 & 169.7 \\
\hline 3 & 1.49 & 2.50 & 90 & 80.2 & 236.5 & 53.3 & 124.8 \\
\hline 4 & 1.82 & 2.50 & 90 & 51.7 & 251.1 & 81.9 & 185.8 \\
\hline 5 & 1.95 & 2.50 & 90 & 67.6 & 282.8 & 57.7 & 108.5 \\
\hline 6 & 2.00 & 2.50 & 90 & 87.3 & 283.2 & 70.9 & 168.1 \\
\hline 7 & 2.10 & 2.50 & 90 & 89.1 & 264.3 & 315.6 & 1147.2 \\
\hline 8 & 2.56 & 2.50 & 70 & 45.5 & 272.1 & 43.5 & 100.9 \\
\hline 9 & 2.74 & 2.50 & 70 & 63.5 & 250.7 & 55.6 & 116.4 \\
\hline 10 & 2.84 & 2.50 & 90 & 86.3 & 278.7 & 77.0 & 251.7 \\
\hline 11 & 3.80 & 2.50 & 90 & 89.0 & 260.1 & 25.5 & 61.6 \\
\hline 12 & 4.60 & 2.50 & 90 & 97.0 & 281.7 & 27.8 & 64.3 \\
\hline 13 & 6.18 & 2.50 & 90 & 121.4 & 293.7 & 44.7 & 87.4 \\
\hline 14 & 6.81 & 2.50 & 90 & 126.5 & 302.4 & 26.5 & 67.8 \\
\hline 15 & 2.23 & Arbitrary & - & 93.3 & 384.2 & 91.3 & 231.1 \\
\hline \hline
\end{tabular}




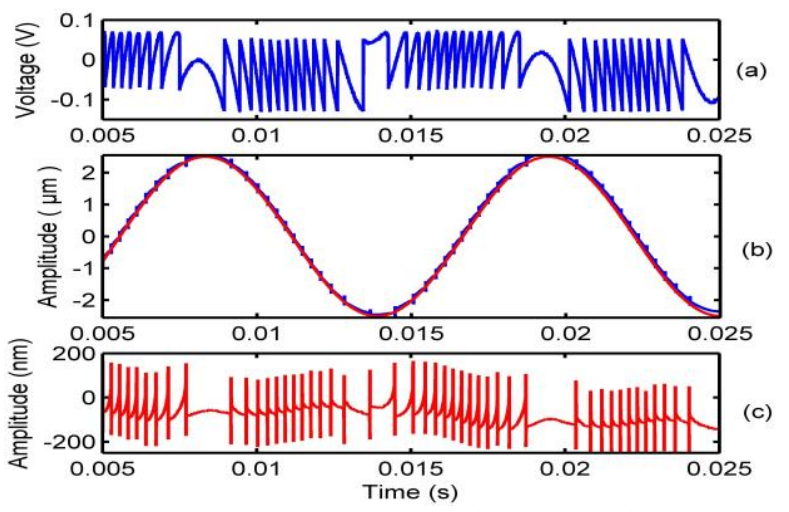

Fig. 13: Harmonic PZT motion using CSU for row 10 of Table I (a): SM signal of estimated $C=2.84$; (b) recovered $D(t)$ using CSU (red) with reference $D(t)$ (blue); (c) error between CSU $D(t)$ and reference PZT $D(t)$.
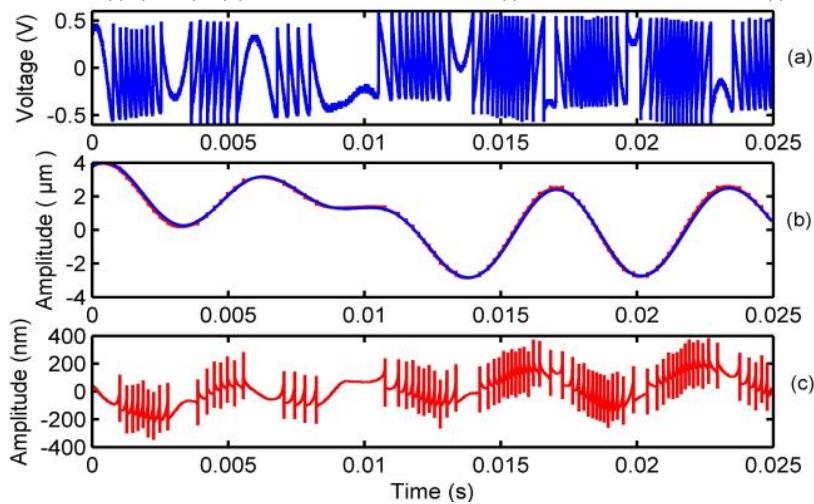

Figure 14: Arbitrary PZT motion using CSU for row 15 of Table I; (a): SM signal of estimated $C=2.23$ (b): Recovered $D(t)$ using CSU (red) with reference $D(t)$ (blue); (c): Error between CSU $D(t)$ and reference PZT $D(t)$.

\section{FPGA BASED HARDWARE DESIGN}

FPGA based hardware design of the CSU algorithm has been performed for a target Virtex6 XC6VLX75T device by using VHDL (VHSIC Hardware Description Language). One thousand samples of simulated normalized SM signal were used in 16 bit fixed point format for verification of our design.

For the target device, it has been found out that CSU algorithm can operate at a sampling frequency of about 265 $\mathrm{MHz}$ with latency of only 3 clock cycles. For same target device, hardware design of PUM has also been executed and tested using the same input SM signal. It has then been found out that PUM can operate at $124.2 \mathrm{MHz}$ of clock frequency with latency of 101316 clock cycles. Timing comparison of CSU and PUM algorithms shows that CSU can execute 1000 samples of SM signal in $3.8 \mu$ s to retrieve target displacement, whereas iterative PUM takes $0.85 \mathrm{~ms}$. So, CSU is more than 200 times faster than PUM for the said case. Power consumption of CSU and PUM has also been computed. The quiescent power consumption is $0.7 \mathrm{~mW}$ with a dynamic supply power of $8 \mathrm{~mW}$ for CSU compared to $77 \mathrm{~mW}$ for PUM.

Comparison of FPGA based design of CSU with PUM (see last columns of Table II and Table III) indicates the vast superiority of CSU (a direct phase unwrapping algorithm) in terms of operating clock frequency, latency, and on-chip resources as compared to classical algorithms [13-16] which are based on analytical solution of excess phase equation [24] using iterative estimation of feedback parameters [26].
TABLE II

FPGA BASED TIMING SUMMARY OF CSU \& PUM

\begin{tabular}{|l|c|c|c|}
\hline \hline & PUM & CSU & $\begin{array}{c}\text { Improvement } \\
\text { Factor }\end{array}$ \\
\hline Maximum clock frequency (MHz) & 124.2 & 265.4 & 2.14 \\
\hline Latency (clock cycles) & 101316 & 3 & 33772 \\
\hline \hline
\end{tabular}

Table III

ON-CHIP RESOURCE UTILIZATION OF CSU \& PUM

\begin{tabular}{|l|c|c|c|}
\hline \hline & PUM & CSU & $\begin{array}{c}\text { Improvement } \\
\text { Factor }\end{array}$ \\
\hline Number of slice registers & 3736 & 74 & 50.48 \\
\hline Number of slice LUTs & 5154 & 149 & 34.59 \\
\hline Number of used DSP48E1s & 3 & 1 & 3 \\
\hline Number of LUT flip-flop pairs & 1398 & 20 & 69.6 \\
\hline \hline
\end{tabular}

As the latency of FPGA based implementation of CSU is only 3 clock cycles while the system clock frequency can be configured up to $265 \mathrm{MHz}$, therefore for an analog-to-digital converter operating at a sampling frequency of $250 \mathrm{M}$ samples per second, the system has an analog bandwidth of $125 \mathrm{MHz}$. As 20 samples are usually required to correctly describe one SM fringe, then for $\lambda_{0}=785 \mathrm{~nm}$, the SM sensor is able to measure maximum speed of $4.9 \mathrm{~m} / \mathrm{s}$. This then means that ultrasonic vibrations can be easily processed. For example, if target vibrations occur at $50 \mathrm{kHz}$, then FPGA based CSU can retrieve such vibrations with p-p amplitude of up to $98.125 \mu \mathrm{m}$. Thus, the designed FPGA based CSU algorithm has the potential to process high bandwidth sensing applications such as ultrasonic vibration characterization of MEMS (microelectro-mechanical system) devices.

\section{DISCUSSION}

Theoretical study and various SM signal results presented in previous sections indicate that CSU provides the minimum displacement reconstruction RMS error for moderate feedback regime SM signals whereas it gives comparatively higher values of RMS error for weak and strong feedback regime SM signals. It can be observed that the reason for higher CSU error in weak feedback regime is the non-linearity of the fringe shape for $C<1$ (see Fig. 2). Likewise, hysteresis, reduced fringe height, and fringe-loss are the reasons for higher CSU error for strong feedback regime. Furthermore, sharp discontinuity at fringe transition location causes an error spike, thus greatly increasing the maximum error at the location of each unwrapped fringe. Analysis and correction of these errors would be pursued in future.

\section{CONCLUSION}

In this paper, a detailed analysis of CSU algorithm has been carried out by theoretically explaining as to how such a simple algorithm provides very good accuracy of about $\lambda_{0} / 10$. Moreover, it recovers displacements with p-p amplitude of < $\lambda_{0} / 2$ making it better than linearization based phase unwrapping algorithms. We also implemented the CSU on FPGA showing that CSU is an efficient real-time approach for SM displacement retrieval utilizing much less time and hardware resources as compared to classical phase unwrapping methods, rendering it attractive for the real-time, embedded, large bandwidth sensing applications. 
Importantly, CSU is able to process all three major feedback regime signals, albeit with varying error performances. However, as demonstrated in [21], it is experimentally possible to robustly stabilize the optical feedback regime (e.g. by using adaptive optics) so that the SM sensor operates within a $C$ range $[1.5,2.5]$ corresponding to the best error performance range of CSU. Such a scheme, then, combines the very fast and low foot print nature of CSU algorithm with very good measurement accuracy.

\section{REFERENCES}

[1] T. Taimre, M. Nikolić, K. Bertling, Y. L. Lim, T. Bosch, and A. D. Rakić, "Laser feedback interferometry: A tutorial on the selfmixing effect for coherent sensing," Advances in Optics and Photonics, vol. 7, pp. 570-631, 2015.

[2] S. Donati, "Developing self-mixing interferometry for instrumentation and measurements," Laser \& Photonics Reviews, vol. 6 , pp. 393-417, 2012.

[3] M. Norgia, G. Giuliani, and S. Donati, "Absolute distance measurement with improved accuracy using laser diode selfmixing interferometry in a closed loop," IEEE transactions on Instrumentation and Measurement, vol. 56, pp. 1894-1900, 2007.

[4] M. Norgia, A. Pesatori, and L. Rovati, "Self-mixing laser Doppler spectra of extracorporeal blood flow: a theoretical and experimental study," IEEE Sensors Journal, vol. 12, pp. 552-557, 2012.

[5] U. Zabit, O. D. Bernal, and T. Bosch, "Design and analysis of an embedded accelerometer coupled Self-Mixing laser displacement sensor," IEEE Sensors Journal, vol. 13, pp. 2200-2207, 2013.

[6] A. Magnani and M. Norgia, "Spectral analysis for velocity measurement through self-mixing interferometry," IEEE Journal of Quantum Electronics, vol. 49, pp. 765-769, 2013.

[7] L. Lu, W. Zhang, B. Yang, J. Zhou, H. Gui, and B. Yu, "Dualchannel self-mixing vibration measurement system in a linear cavity fiber laser," IEEE Sensors Journal, vol. 13, pp. 4387-4392, 2013.

[8] G. Giuliani, S. Bozzi-Pietra, and S. Donati, "Self-mixing laser diode vibrometer," Measurement Science and Technology, vol. 14, p. 24, 2002 .

[9] Z. A. Khan, U. Zabit, O. D. Bernal, M. O. Ullah, and T. Bosch, "Adaptive Cancellation of Parasitic Vibrations Affecting a SelfMixing Interferometric Laser Sensor," IEEE Transactions on Instrumentation and Measurement, vol. 66, pp. 332-339, 2017.

[10] O. D. Bernal, H. C. Seat, U. Zabit, F. Surre, and T. Bosch, "Robust Detection of Non-Regular Interferometric Fringes From a SelfMixing Displacement Sensor Using Bi-Wavelet Transform," IEEE Sensors Journal, vol. 16, pp. 7903-7910, 2016.

[11] A. L. Arriaga, F. Bony, and T. Bosch, "Real-time algorithm for versatile displacement sensors based on self-mixing interferometry," IEEE Sensors Journal, vol. 16, pp. 195-202, 2016.

[12] U. Zabit, T. Bosch, and F. Bony, "Adaptive transition detection algorithm for a self-mixing displacement sensor," IEEE Sensors Journal, vol. 9, pp. 1879-1886, 2009.

[13] S. Merlo and S. Donati, "Reconstruction of displacement waveforms with a single-channel laser-diode feedback interferometer," IEEE Journal of Quantum Electronics, vol. 33, pp. 527-531, 1997.

[14] C. Bes, G. Plantier, and T. Bosch, "Displacement measurements using a self-mixing laser diode under moderate feedback," IEEE Transactions on Instrumentation and Measurement, vol. 55, pp. 1101-1105, 2006

[15] Y. Fan, Y. Yu, J. Xi, and J. F. Chicharo, "Improving the measurement performance for a self-mixing interferometry-based displacement sensing system," Applied Optics, vol. 50, pp. 50645072, 2011

[16] O. D. Bernal, U. Zabit, and T. Bosch, "Study of laser feedback phase under self-mixing leading to improved phase unwrapping for vibration sensing," IEEE Sensors Journal, vol. 13, pp. 4962-4971, 2013.
[17] S. Donati and M. T. Fathi, "Transition From Short-to-Long Cavity and From Self-Mixing to Chaos in a Delayed Optical Feedback Laser," IEEE Journal of Quantum Electronics, vol. 48, pp. 13521359, 2012.

[18] U. Zabit, F. Bony, T. Bosch, and A. D. Rakic, "A self-mixing displacement sensor with fringe-loss compensation for harmonic vibrations," IEEE Photonics Technology Letters, vol. 22, pp. 410$412,2010$.

[19] S. Ottonelli, F. De Lucia, M. di Vietro, M. Dabbicco, G. Scamarcio, and F. P. Mezzapesa, "A compact three degrees-offreedom motion sensor based on the laser-self-mixing effect," IEEE Photonics Technology Letters, vol. 20, pp. 1360-1362, 2008.

[20] U. Zabit, O. Bernal, and T. Bosch, "Self-mixing sensor for realtime measurement of harmonic and arbitrary displacements," in Instrumentation and Measurement Technology Conference (I2MTC), 2012 IEEE International, 2012, pp. 754-758.

[21] O. D. Bernal, U. Zabit, and T. M. Bosch, "Robust method of stabilization of optical feedback regime by using adaptive optics for a self-mixing micro-interferometer laser displacement sensor," IEEE Journal of Selected Topics in Quantum Electronics, vol. 21, pp. 336-343, 2015.

[22] M. Norgia and A. Pesatori, "Fully analog self-mixing laser vibrometer," in Instrumentation and Measurement Technology Conference (I2MTC), 2011 IEEE, 2011, pp. 1-4.

[23] U. Zabit, O. Bernal, and T. Bosch, "Time-frequency signal processing for a Self-Mixing laser sensor for vibration measurement," in Sensors, 2012 IEEE, 2012, pp. 1-4.

[24] R. Lang and K. Kobayashi, "External optical feedback effects on semiconductor injection laser properties," IEEE Journal of Quantum Electronics, vol. 16, pp. 347-355, 1980.

[25] T. Taimre and A. D. Rakić, "On the nature of Acket's characteristic parameter C in semiconductor lasers," Applied Optics, vol. 53, pp. 1001-1006, 2014.

[26] J. Xi, Y. Yu, J. F. Chicharo, and T. Bosch, "Estimating the parameters of semiconductor lasers based on weak optical feedback self-mixing interferometry," IEEE Journal of Quantum Electronics, vol. 41, pp. 1058-1064, 2005.

[27] A. Magnani, A. Pesatori, and M. Norgia, "Self-mixing vibrometer with real-time digital signal elaboration," Applied Optics, vol. 51, pp. 5318-5325, 2012.

[28] Y. Tao, M. Wang, and D. Guo, "Compound cavity theory of resonant phase modulation in laser self-mixing ultrasonic vibration measurement," Optical Engineering, vol. 55, pp. 074107-074107, 2016.

[29] G. Plantier, C. Bes, and T. Bosch, "Behavioral model of a selfmixing laser diode sensor," IEEE Journal of Quantum Electronics, vol. 41, pp. 1157-1167, 2005.

[30] O. D. Bernal, U. Zabit, and T. Bosch, "Classification of laser selfmixing interferometric signal under moderate feedback," Applied Optics, vol. 53, pp. 702-708, 2014.

[31] Y. Yu, J. Xi, J. F. Chicharo, and T. M. Bosch, "Optical feedback self-mixing interferometry with a large feedback factor: behavior studies," IEEE Journal of Quantum Electronics, vol. 45, pp. 840848, 2009.

[32] A. Arasanz, F. Azcona, S. Royo, A. Jha, and J. Pladellorens, "A new method for the acquisition of arterial pulse wave using selfmixing interferometry," Optics \& Laser Technology, vol. 63, pp. 98-104, 2014. 